\title{
Rabaska
}

Revue d'ethnologie de l'Amérique française

FRENETTE, YVES et FRANCE MARTINEAU en collaboration avec

VIRGIL BENOIT. Les Voyages de Charles Morin, charpentier canadien-français. Québec, Presses de l'Université Laval, « Les Voies du français », 2018, X-566 p. ISBN 978-2-7637-3879-6

\section{Gabriel Martin}

Volume 18, 2020

URI : https://id.erudit.org/iderudit/1072935ar

DOI : https://doi.org/10.7202/1072935ar

Aller au sommaire du numéro

Éditeur(s)

Société québécoise d'ethnologie

ISSN

1703-7433 (imprimé)

1916-7350 (numérique)

Découvrir la revue

Citer ce compte rendu

Martin, G. (2020). Compte rendu de [FrenetTe, Yves et FranCE MARTineAu en collaboration avec VIRGIL BENOIT. Les Voyages de Charles Morin,

charpentier canadien-français. Québec, Presses de l'Université Laval, " Les

Voies du français », 2018, x-566 p. ISBN 978-2-7637-3879-6]. Rabaska, 18,

346-349. https://doi.org/10.7202/1072935ar d'utilisation que vous pouvez consulter en ligne. 
les textes rassemblés permettraient de constater « la dispersion [...] de ceux qui [...] pensent » (p. 288) le patrimoine. Le pouvoir, auparavant détenu par certains, semble devenir « le pouvoir de tous », en raison notamment de la multiplication des objets de patrimoine, des échelles où ce patrimoine est présent, puis des « va-et-vient juridiques » (p. 288) entre ces mêmes échelles.

Accessibles, variés et encadrés par une introduction qui pose efficacement les paramètres de la réflexion ainsi que par une conclusion qui propose des pistes de réflexion riches, les textes rassemblés ici ne manqueront pas de susciter l'intérêt de ceux qui étudient le patrimoine tout comme, à n'en pas douter, de ceux qui œuvrent dans le domaine ou encore qui entretiennent simplement un intérêt pour celui-ci. Cela dit, aussi intéressantes que soient les diverses contributions de cet ouvrage, il nous apparait que le plus grand mérite de ce dernier réside dans la proposition et les questionnements qui se dégagent de l'ensemble. Les questions théoriques et pratiques ainsi que les tensions explorées appellent en effet à repenser le champ patrimonial, au niveau des conceptions ou des modes de désignation et de gestion, par exemple, ce qui amène même Morisset à s'interroger sur l'institutionnalisation du patrimoine et à réfléchir sur l'émancipation de ce dernier. En effet, le patrimoine « ne se définit[-il pas] que par le discours performatif de ceux qui mobilisent le mot et ses déclinaisons » (p. 291)?

Myriam Mathieu-BédARd Université du Québec à Montréal

Frenette, Yves et France Martineau en collaboration avec Virgil Benoit. Les Voyages de Charles Morin, charpentier canadien-français. Québec, Presses de l'Université Laval, «Les Voies du français », 2018, x-566 p. ISBN 978-2-7637-3879-6.

Ce livre présente une édition critique des mémoires de Charles Morin (1849-1922), un charpentier d'origine canadienne-française qui a voyagé pendant plus d'une décennie en Amérique du Nord avant de se fixer aux États-Unis. L'ouvrage est divisé en trois parties : une introduction générale, une note sur l'établissement du texte et la transcription des mémoires à proprement parler.

L'introduction, rédigée par Yves Frenette et France Martineau, en collaboration avec Virgil Benoit, expose d'entrée de jeu l'intérêt des mémoires de Morin qui offrent selon eux « un accès privilégié à la construction et à l'évolution de l'identité d'un Canadien français, ainsi qu'un aperçu de la langue parlée à son époque » et qui « éclairent sur divers aspects de la vie socio-économique en Amérique du Nord à l'ère industrielle, notamment 
sur la centralité des migrations » (p. 1). Les auteurs fournissent ensuite les informations nécessaires à la critique externe des manuscrits, dont l'histoire de leur préservation, leur datation incertaine (qui se situerait entre 1894 et 1916), leur description matérielle et leur contexte probable de réalisation.

Par la suite, ils dépeignent l'univers familial dans lequel a grandi le mémorialiste et tracent les grandes lignes de son parcours, en s'appuyant entre autres sur de la documentation généalogique. Ils nous apprennent que Morin est issu d'une famille nombreuse et peu nantie, qu'il a fréquenté l'école jusqu'à 11 ans et qu'il n'y retourne que quelques mois à l'âge de 15 ans, ce qui lui permet d'acquérir les rudiments de la lecture et de l'écriture. Vivant à une époque de forte mobilité géographique, il entame ses pérégrinations quelques années plus tard. Il migre alors fréquemment de localité en localité, parfois sur de longues distances, non seulement pour trouver du travail, mais aussi par esprit d'aventure. Jusqu'à l'âge de 35 ans, il se déplace d'un coin à l'autre du Canada et des États-Unis. En 1884, il s'établit définitivement à Argyle, au Minnesota, où il se forge une avantageuse réputation comme charpentier et s'engage dans les affaires paroissiales.

Les auteurs observent que Morin a constitué ses mémoires en relatant des évènements précis de sa vie, sur lesquels il livre des réflexions personnelles et souvent moralisantes, vraisemblablement destinées à son cercle familial. Ils remarquent que sa difficile quête de travail et sa résistance devant l'adversité constituent un fil conducteur des récits. Ils soulignent aussi les grands traits de l'identité de Morin, qui se représente comme un "Canadien », et qui pourrait plus précisément être décrit comme un francophone originaire de la vallée du Saint-Laurent adhérant fortement à l'idéologie catholique ambiante.

Une section de l'introduction, qui s'appuie largement sur les travaux de la linguiste France Martineau, s'attarde à analyser certaines caractéristiques de la langue employée dans les mémoires. On y voit que Morin recourt à de nombreuses ressources linguistiques caractéristiques de la langue orale familière, car il maîtrise peu les normes du code écrit. Parmi les divers faits observés, notons des emprunts non normatifs à l'anglais (p. ex. boss, foreman et factrie), des graphies phonétisantes (p. ex. juillette, charcher et besenismans pour juillet, chercher et businessman) ou encore des emplois grammaticaux aujourd'hui jugés non standards (p. ex. je vas pour je vais).

Vient ensuite le cœur de l'ouvrage, qui est constitué par les mémoires de Morin. Les manuscrits, qui existent en deux versions distinctes, sont intégralement retranscrits dans le livre, en conformité avec les originaux. En regard de chaque retranscription, une transposition orthographique normalisée est fournie pour faciliter le décodage du texte par un lectorat contemporain.

Les principes d'établissement du texte et de normalisation orthographique sont exposés en détail par Martineau, qui s'est acquittée de leur mise en œuvre. 
Dans la version normalisée, la chercheuse a essentiellement régularisé les graphies, mais a préservé la grande majorité des éléments grammaticaux ou lexicaux qui s'écartent de la norme contemporaine la plus communément admise. L'orthographe française n'étant que partiellement phonétique ou phonémique, certaines variantes de prononciation utilisées par Morin s'en trouvent nécessairement gommées, mais le texte acquiert grandement en lisibilité. Par ailleurs, les crochets et l'italique sont amplement mis à profit pour faciliter encore plus le décodage. Les désavantages éventuellement causés par la transposition normalisée du texte sont entièrement compensés par la présence de l'original.

Morin a découpé ses mémoires en 25 épisodes de tailles variables. Les auteurs respectent ce découpage et font précéder chaque épisode d'une introduction qui en présente et commente le contenu. Aussi bien ces textes de présentation que les épisodes eux-mêmes sont abondamment annotés. Les références documentaires et les commentaires fournis sont utiles. Nous savons notamment gré aux auteurs d'être parvenus à éclairer le sens de mots en chinook, une langue amérindienne de la côte ouest que Morin cite par endroit sans nécessairement donner de précisions explicites.

La présentation du livre est soignée et répond aux normes de publication universitaires. La composition typographique (principalement effectuée en Times New Roman et en Myriad) est plutôt conventionnelle, mais sobre et équilibrée. Les reproductions photographiques sont de qualité. Signalons la commodité de la carte imprimée à la page 11, qui aide à mieux visualiser les déplacements de Morin au fil des années.

En revanche, nous estimons que l'éditeur aurait pu peaufiner certains aspects, certes mineurs, qui relèvent surtout de la mise en pages. Ainsi, la synthèse visuelle qui remplit la page 19 aurait à notre avis gagné à être scindée ou simplifiée, la présence de lignes verticales dans un tableau et le fait qu'il déborde des marges étant souvent un indice qu'il pourrait être amélioré. De même, l'intitulé « Sources », présent dans certaines notes (p. ex. en p. 92) aurait préférablement été placé en retrait. On relève aussi quelques rares coquilles, presque inévitables dans un document d'une telle ampleur (p. ex. l'emploi d'une police trop petite sur un caractère du mot et en p. 22 ; l'absence d'italique sur l'emploi autonymique de -er et -é en p. 30 ; la substitution de l'abréviation $m s$ par $m$ en p. 31). Enfin, l'ajout d'une table des abréviations conventionnelles (p. ex. l., ms, p. pour ligne, manuscrit et page), sans être essentielle, aurait été pratique pour les lecteurs moins chevronnés. En toute justice, soulignons que ces détails demeurent négligeables et n'entachent pas réellement la lecture d'un livre somme toute bien conçu.

Une des forces les plus saillantes de l'ouvrage concerne, à notre avis, l'attention consacrée aux questions en lien avec la langue. La courte analyse 
linguistique présentée en introduction est convaincante dans l'ensemble. De plus, le travail de transcription et de normalisation orthographique nous apparaît exemplaire. Nous souhaitons à cet égard que l'ouvrage fasse des émules et qu'il serve de prélude à l'édition d'autres archives familiales anciennes produites par des personnes peu lettrées dont les écrits fournissent bien souvent des données profitables aux linguistes. Nous abondons ainsi dans le sens de Martineau, qui affirme « espérer que plus de textes de cette nature seront édités, de façon à faire entendre la langue de l'époque dans toutes ses nuances $\gg$ (p. 54).

$\mathrm{Au}$ demeurant, les ethnologues trouveront quelques informations liées à leur champ d'expertise dans cet ouvrage. Les réflexions subjectives et les observations de Morin sur certains membres des Premiers Peuples qu'il a rencontrés, comme celles qui se trouvent dans l'épisode 11 sur « Les mœurs des Sauvages et leurs coutumes » (p. 391-415), ont de bonnes chances d'attiser leur intérêt.

En somme, cet ouvrage représente un travail patient et professionnel de valorisation documentaire. En plus d'être de lecture agréable, il fournit notamment du matériel linguistique de première main utile à l'étude du français nord-américain d'autrefois.

Gabriel Martin

Université de Sherbrooke

Gallichan, Gilles. L'Église Saint-Charles de Limoilou. Témoin d'histoire et chantier d'avenir. Photographies de MARC LaJoIE. Québec, Éditions du Septentrion, 2020, 238 p. ISBN 978-2-8944-8955-0.

L'église Saint-Charles de Limoilou, située dans le quartier du même nom, au sein de l'arrondissement La Cité-Limoilou, figure au nombre des bâtiments religieux de Québec qui font actuellement l'objet d'un processus de conversion. Fort souvent, la conversion des propriétés conventuelles et, à une échelle différente, des églises se traduit par des projets d'habitation d'ampleurs variables. Or, à cet égard, la particularité de la conversion de l'église Saint-Charles de Limoilou réside probablement dans le fait qu'elle est plutôt alimentée par un mouvement visant le développement d'un laboratoire d'innovations sociales nommé La FABRIQC. Teinté par les principes et la philosophie du développement durable, ce laboratoire est tourné vers des initiatives citoyennes menées en co-construction.

On pourrait être porté à croire que la publication d'un ouvrage concernant l'église Saint-Charles à ce moment-ci puisse s'inscrire, d'une façon ou d'une autre, dans le contexte de la conversion du bâtiment, dans la mesure 